\title{
Scheduling of two and three machine robotic cells with fuzzy methodology
}

\author{
M. Hossein Fazel Zarandi *, M. Mehdi Fazel Zarandi, M. Yousef Maknoon, J. Masoumi \\ Department of Industrial Engineering, Amir Kabir University of Technology, Tehran, Iran, P.O. Box: 15875-4413
}

(Received March 6 2007, Accepted August 28 2007)

\begin{abstract}
This paper addresses the scheduling of robotic cells with two and three machines with fuzzy methodology. Some of the scheduling problems of these categories are transferred into a solvable traveling salesman problem that can be solved in a polynomial time. For generating the optimal part sequencing in the cells, the Gilmore and Gomory algorithm is modified and instead, a fuzzy Gilmore and Gomory algorithm is developed. Then, the proposed algorithm is tested and verified in a supplier company that produces part for an automobile industry. In any case, we compare the results of the proposed fuzzy method with those of crisp ones. The results show the superiority of the proposed algorithm in terms of flexibility, robustness, and reduction of cycle times.
\end{abstract}

Keywords: fuzzy scheduling, fuzzy robotic cells, fuzzy cycle time, fuzzy numbers, fuzzy traveling salesman problem

\section{Introduction}

Industrial robots play an important role in advanced manufacturing systems. The most typical anthropomorphic, or humanlike, characteristic of a robot is its arm. The arm, together with the robotics' capacity to be programmed, makes it ideally suited to a variety of production tasks, including machine loading, spot welding, spray painting, and assembly. A robot can be programmed to perform a sequence of mechanical motions, and can repeat that motion sequence over and over until reprogrammed to perform some other sequence ${ }^{[9]}$.

The problem of deciding how to form parts and machines into manufacturing cells has been studied by many authors, including McCauley ${ }^{[19]}$, Burbidge ${ }^{[3]}$, Rajagopalan and Batra ${ }^{[23]}$, King and Spachis ${ }^{[14]}$, Chan and Milner ${ }^{[4]}$, Seifoddini and Wolfe ${ }^{[24]}$, Kusiak ${ }^{[17]}$, and Askin and $\mathrm{Chiu}^{[1]}$. Hall et al. ${ }^{[10]}$ suggest heuristic procedures of a clustering type for forming the cells. The main focus, however, is on problems of scheduling within cells rather than of cell formation. Although manufacturing cells can have human operators to perform material handling, the full benefits to productivity form the use of cells are only realized by robot handling. In such a case, the manufacturing cell is known as a robotic cell. Hartley ${ }^{[12]}$ points out that a cell system

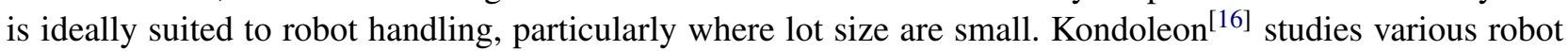
assembly system configurations and their effect on output rate. Baumann et al. ${ }^{[2]}$ study robot and machine utilization in a robotic cell. Simulation studies of robotic cells are described by Claybourn and Hewitt ${ }^{[5]}$, and Noh and Herring ${ }^{[21]}$. Drezner and Nof ${ }^{[6]}$ design heuristics for various operations in an assembly cell. Wilhelm and Sarin ${ }^{[27]}$ consider the parts scheduling problem in robotic cells with various configurations, including parallel identical and no identical machines, and flow line manufacturing. They, however, did not present any optimal scheduling policy for the cells. Nof and Drezner ${ }^{[20]}$ discuss the assembly cell taking into account the necessary operations and movements. A heuristic solution is proposed and tested in their work.

The computational complexity of several scheduling problem in assembly cells with one or two robots is discussed by Wilhelm ${ }^{[26]}$. R. Obinowitz et al. ${ }^{[22]}$ formulate a mixed integer model for a variety of objective

\footnotetext{
*E-mail address: zarandi@aut.ac.ir; mmfazel@yahoo.com; j_masoumi7@yahoo.com; yousef@maknoun.com.
} 


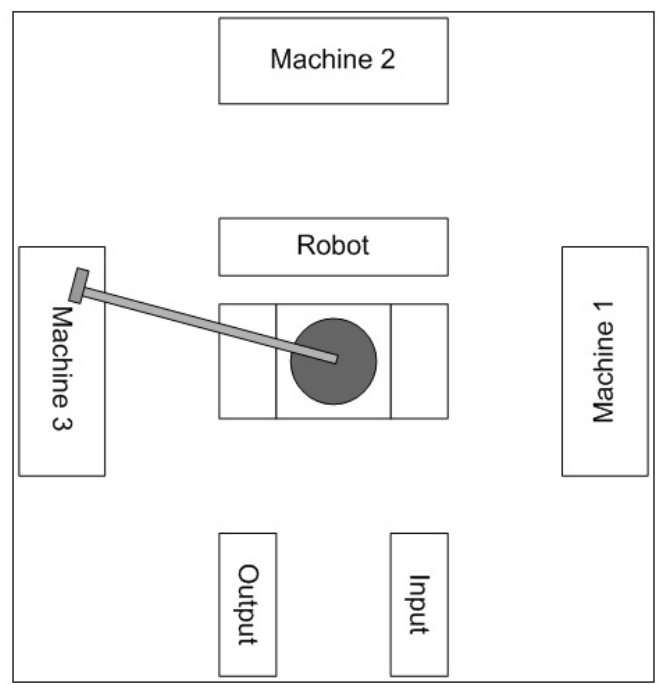

Fig. 1. Robotic cell with three machines

function in a multi-robot machining and assembly cell. Unfortunately, the size of their formulation increases very rapidly with the size of the cell. Longendran and Sriskandarajah ${ }^{[18]}$ study three different robotic cell layouts: the robot-centered, the mobile robot cell, and the in-line robot cell. For a given robot move sequence, they identify part sequence for a minimal part set (MPS) that minimizes the cycle time, or equivalently maximizes the long run production rate. Kise et al. ${ }^{[15]}$ study the makespan version of the part sequencing problem under a specified move cycle, and present a polynomial time algorithm. Sethi et al. attempt to minimize the cycle time of an MPS. They find the optimal sequence of moves for a two-machine robot centered cell producing a single part type. For the three machine case they identify the six potentially optimal robot move cycles that produce one unit and show, under specific assumptions about the data, that two are dominated by the other four. They, then, provide sufficient conditions under which each of the four no dominated cycles is optimal. Kamoun et al. ${ }^{[13]}$ describe and test simple heuristic procedures for the part sequencing problem in several cell configurations, where the problem is intractable. They also study how to design robotic cells for efficient performance by grouping machines into cells, by identifying good part sequences, and by providing appropriate size buffers between cells, in a larger manufacturing system.

Kamoun ${ }^{[13]}$ study a robotic cell containing m machines for any constant $m \leq 2$. The part sequencing problems associated with a given robot move cycles for producing one unit are classified as follows:

(i). Sequence independent;

(ii). Formulated as a traveling salesman problem;

(iii). Unary NP-hard but not having TSP structure.

Some of the problems in case (ii) are special cases which can be solvable in polynomial times. In this classification, they prove that the part sequencing problem associated with exactly $2 m-2$ of the $m$ ! Potentially optimal one-unit cycles are polynomial solvable, where $m$ is the number of machines. The remaining cycles have associated part-sequencing problems which are unary NP-hard.

Hall et al. ${ }^{[11]}$ show with an example that the best one-unit cycle can be suboptimal in two machine cells producing multiple part type, and provide an efficient algorithm (MINICYCLE) to find optimal robot move cycle. They also show, through the solution of a mixed integer program, that a policy of repeating one-unit cycle dominates much more complicated cycles producing two-units. In three machines, they study the ways in which a robotic cell converges to the steady state. A steady state is a condition within a cell in which the waiting time of the robot, for each part on each machine, is identical to its value in the previous Minimal Part Set (MPS). Hall et al. ${ }^{[9]}$ study the complexity and steady state analysis in scheduling robotic cell and prove that in two out of six potentially optimal robot move cycle (S2, S6) producing one-unit, the recognition version of the part sequencing problem are NP-Complete.

All of the above research assumes that the processing times of the parts on machines, the traveling times of robot between machines and the cycle times of the different schedules to be deterministic. In real world 
problems, this assumption is not adequate. In this paper, we use fuzzy numbers to make the robotic scheduling problems more realistic and robust.

The rest of the paper is organized as follows: Section 2 presents cyclic scheduling. Section 3 presents the main objective of Gilmore and Gomory algorithm. In section 4, the fuzzy cycle times in robotic cells with 2 and 3 machines are described. Section 5 presents fuzzy arithmetic that is used to calculate the fuzzy cycle time and method for de-fuzzifing it. In section 6, the steps of "Fuzzy Gilmore and Gomory algorithm" are described. In section 7 numerical examples are generated and the results of fuzzy and crisp cycle times are compared. Finally, in section 8, conclusions and future works are presented.

\section{Cyclic scheduling}

In general, scheduling problems can be classified as: flow shops, Job shops, and open shops. From them, flow shops are the simplest problems. However, most of the flow shops scheduling problems are either NPComplete or NP-hard. Among different kinds of the flow shops, two of them are more prevalent in the real world problems: No-Wait and Blocking. In No-Wait flow shops, a job is not allowed to remain on a machine or between machines after finishing its process on that machine. So, after finishing the process of a job on a machine, it should move to the next machine immediately. In blocking flow shops, the jobs are allowed to remain on a machine after their process are finished on that machine, because there is not any buffer available between machines. A special case of flow shops is cyclic one. In a cyclic flow shop, the scheduling is repeated based on minimum part set (MPS). Fig. 2 and 3 show the No-Wait and Blocking cyclic scheduling with two machines.

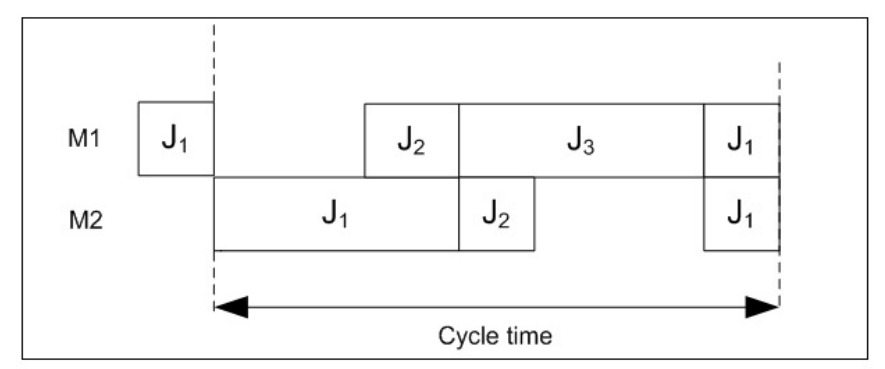

Fig. 2. A No-Wait cyclic flow shop

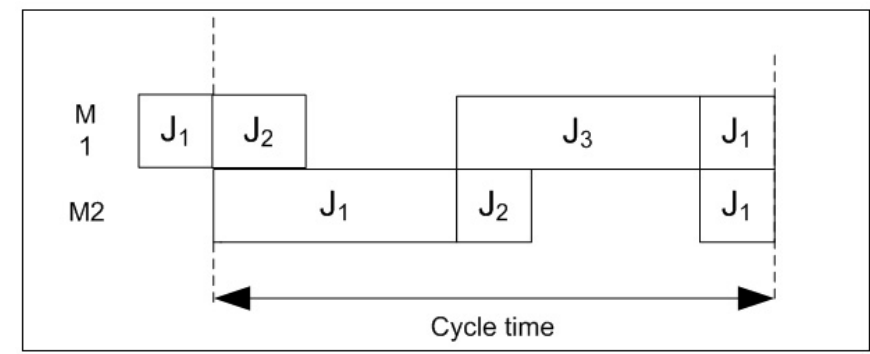

Fig. 3. A blocking cyclic flow shop

Hall et al. ${ }^{[9]}$ show that in a two-machine cyclic scheduling a no-wait problem can be transferred into blocking problem and vise versa.

\section{Gilmore and Gomory algorithm}

Gilmore and Gomory ${ }^{[7]}$ consider the sequencing problem for $\mathrm{N}$ jobs on a machine having a state described by a single real variable $X$. Jobs $J_{1}, J_{2} \cdots J_{N}$ are to be processed on the machine in some order. Each 
job has two associated numbers $\mathrm{Ai}$ and $\mathrm{Bi}$. To start the $i^{t h}$ job, the machine must be in state $A_{j}$, i.e., $X=A_{j}$ and at the completion of the $i^{t h}$ job the machine state is automatically $B_{i}$. If $J_{j}$ is to follow $J_{i}$, the state of the machine must be changed to $A_{j}$. The cost of this change, i.e., the cost of having job $j$ follow job $i$ is $C_{i j}$. This can be calculated as follows:

$$
\begin{array}{llll}
C_{i j}=\int_{B_{i}}^{A_{j}} f(x) d x & \text { if } & A_{j} \geq B_{i} \\
C_{i j}=\int_{B_{i}}^{A_{j}} g(x) d x & \text { if } & B_{i}>A_{j}
\end{array}
$$

where, $f(x)$ and $g(x)$ are any integrable functions satisfying $f(x)+g(x)>0 . f(x)$ can be interpreted as the cost density for increasing the state variable and $g(x)$ the cost density for decreasing it.

This problem is of course closely connected to Traveling Salesman Problem (TSP). To see this, let $J_{i}$ plays the role of nodes or cities, and $C_{i j}$ be the cost of going from node $(i)$ to node $(j)$. We are looking for the shortest path that passes once through each node. The traveling salesman looks for the shortest path that passes once through each node and ends up at the starting point. It looks for the shortest tour.

The Gilmore and Gomory's problem becomes the tour problem if the machine is assumed to be in a state $\mathrm{B} 0$ at the start, before the jobs are run, and is required to be left in a state $A_{0}$ at the end after all $\mathrm{N}$ jobs are done. Specifically, if we add a new job $J_{0}$, there is always one-to-one correspondence between the tours $J_{0} J_{i 1} J_{i 2} \cdots J_{i N} J_{0}$ of the enlarged problem and the sequence $J_{i 1} J_{i 2} \cdots J_{i N}$ of the original problem, so one can minimize over tours, and then, dropping $J_{0}$, have the least sequence. By this algorithm they give a solution requiring only $O(N 2)$ simple steps. This solution permits a characterization of those directed graphs of a special class possess Hamiltonian circuits.

\section{Fuzzy cycle time in robotic cells with 2 and 3 machine}

Sethi et al. ${ }^{[25]}$ discuss the cycle times in robotic cell with 2 and 3 machines. We generalize their model into fuzzy cycle time robotic cell. Moreover, we also generalize their crisp notation into fuzzy ones as follows: $M$ : the number of machines in the cell (here 2 or 3 ).

$P_{1}, P_{2}, \cdots, P_{K}$ : the part type to be produced.

$r_{1}, r_{2}, \cdots, r_{k}$ : The minimal ratios of parts to be produced.

MPS: a minimal part set.

$n=r_{1}+r_{2}+\cdots+r_{k}$ : the total number of part.

$\tilde{\theta}_{i}, \tilde{\lambda}_{i}, \tilde{\varphi}_{i}$ : Fuzzy processing times of part $i$ on machine $M_{1}, M_{2}, M_{3}$ respectively.

$\tilde{\delta}_{0}$ : Fuzzy time taken by additional linear robot movement when traveling between two consecutive machines $M_{i}$ and $M_{i+1}$ after rotational movement has been completed.

$\tilde{\delta}_{i}$ : The fuzzy time taken by a rotational robot movement when traveling between two consecutive machine $M_{i-1}$ and $M_{i}, 1<i<m+1$.

$\tilde{\varepsilon}_{1}$ : The fuzzy time taken by the robot to pick up a part at $\mathrm{i}$.

$\tilde{\varepsilon}_{2 i}$ : The fuzzy time taken by the robot to load a part on to machine $M_{i}$.

$\tilde{\varepsilon}_{2 i+1}$ : The fuzzy time taken by the robot to unload a part from machine $M_{i}$.

$\tilde{\varepsilon}_{2 m+1}$ : The fuzzy time taken by the robot to drop a part at $\mathrm{O}$.

$\tilde{\gamma}$ : The fuzzy time saved by not stopping at an intermediate point during a robot movement between two nonconsecutive machines.

$\tilde{W}_{j}^{i}$ : The fuzzy waiting time of job $i$ on machine $j$.

The standard classification scheme for scheduling problems can be denoted $\Psi_{1}\left|\Psi_{2}\right| \Psi_{3}$, where $\Psi_{1}$ indicates the scheduling environment, $\Psi_{2}$ describes the job characteristics or restrictive requirements and $\Psi_{3}$ defines the objective function to be minimized $\left(\right.$ Graham $\left.^{[8]}\right)$.

For $\Psi_{1}$

$\mathrm{MRC}_{m}$ : a mobile -robot cell with m machine.

$\mathrm{RCC}_{m}$ : a robot-centered cell with m machine.

$\mathrm{IRC}_{m}$ : an in-line cell with $\mathrm{m}$ machine.

For $\Psi_{2}$

$\mathrm{K}=$ the number of part0-types. 
R-unit=the problem is being solved over robot move cycle that produce $\mathrm{r}$ units.

$S_{i}=$ robot move cycle $S_{i}$ alone is used.

$\delta_{i}=\delta=$ the travel time between any pair of consecutive machine is equal.

$\epsilon_{i}=\varepsilon$ : the load and unload times at all machine are equal.

For $\Psi_{3}$

$C_{t}=$ the average steady state cycle time for the repetitive manufacturing of an MPS.

$C_{\max }=$ the make span for the manufacturing.

\subsection{Fuzzy cycle times in two-machine-multiple-part type robotic cells}

As shown in Fig. 4, there are two possible robot move cycles $S_{1}$ and $S_{2}$ for problem $\mathrm{MRC}_{2}|k>2| C_{t}$ (Sethi et al. ${ }^{[25]}$ ). The fuzzy cycle time for robot move cycle $S_{1}$ is:

$$
\tilde{T}_{1(\sigma)}=n \tilde{\rho}+\sum_{i=1}^{n}\left(\tilde{\theta}_{i}^{\prime}+\tilde{\lambda}_{i}^{\prime}\right)
$$

Where

$$
\begin{aligned}
& \tilde{\theta}_{i}^{\prime}=\tilde{\theta}_{i}+\tilde{\varepsilon_{1}}+\tilde{\varepsilon_{2}}+2 \tilde{\delta_{2}}-\tilde{\gamma} \quad \text { For } \quad i=1,2, \cdots, n \\
& \tilde{\lambda_{i}^{\prime}}=\tilde{\lambda_{i}}+\tilde{\varepsilon_{5}}+\tilde{\varepsilon_{6}}+2 \tilde{\delta_{3}}-\tilde{\gamma} \quad \text { For } i=1,2, \cdots, n \\
& \tilde{\mu}=2 \sum_{I=0}^{3} \tilde{\delta}_{i}+\tilde{\varepsilon_{1}}+\tilde{\varepsilon_{2}}+\tilde{\varepsilon_{5}}+\tilde{\varepsilon_{6}}-2 \tilde{\gamma} \\
& \tilde{\rho}=\tilde{\varepsilon_{3}}+\tilde{\varepsilon_{4}}+2 \tilde{\delta_{0}}+2 \tilde{\delta_{2}}
\end{aligned}
$$

The cycle time for robot move cycle $S_{2}$ is:

$$
\tilde{T}_{2(\sigma)}=n \tilde{\rho}+\sum_{i=1}^{n} M A X\left(\tilde{\mu}, \tilde{\theta}_{\sigma(i)}{ }^{\prime}, \lambda_{\sigma(i+1)}{ }^{\prime}\right)
$$

The part sequencing problem, under robot move cycle $S_{2}$ can be transformed into the problem $F_{2} \mid$ no wait $\mid \tilde{C} t$, having the form: minimize $\left(C+\sum \max \left(\tilde{e}_{\sigma(i+1)}, \tilde{f_{\sigma(i)}}\right)\right)$, where $C$ is a constant and $\tilde{e}_{\sigma(i)}=\tilde{a}_{\sigma(i)}^{\prime}$ and $f_{\tilde{\sigma}(i)}=\max \left\{\mu, \tilde{b}_{\sigma(i)}^{\prime}\right\}$ are the fuzzy processing times for part $P_{\sigma(i)}$ on the first machine $\left(M_{1}^{\prime}\right)$ and the second machine $\left(M_{2}^{\prime}\right)$, respectively. In this problem, the operations of any job are performed continuously without waiting on or between machines. We recall that this form of problem can be solved by Gilmore and Gomory algorithm. Of course, when all times are fuzzy we should fuzzify Gilmore and Gomory algorithm ${ }^{[7]}$.
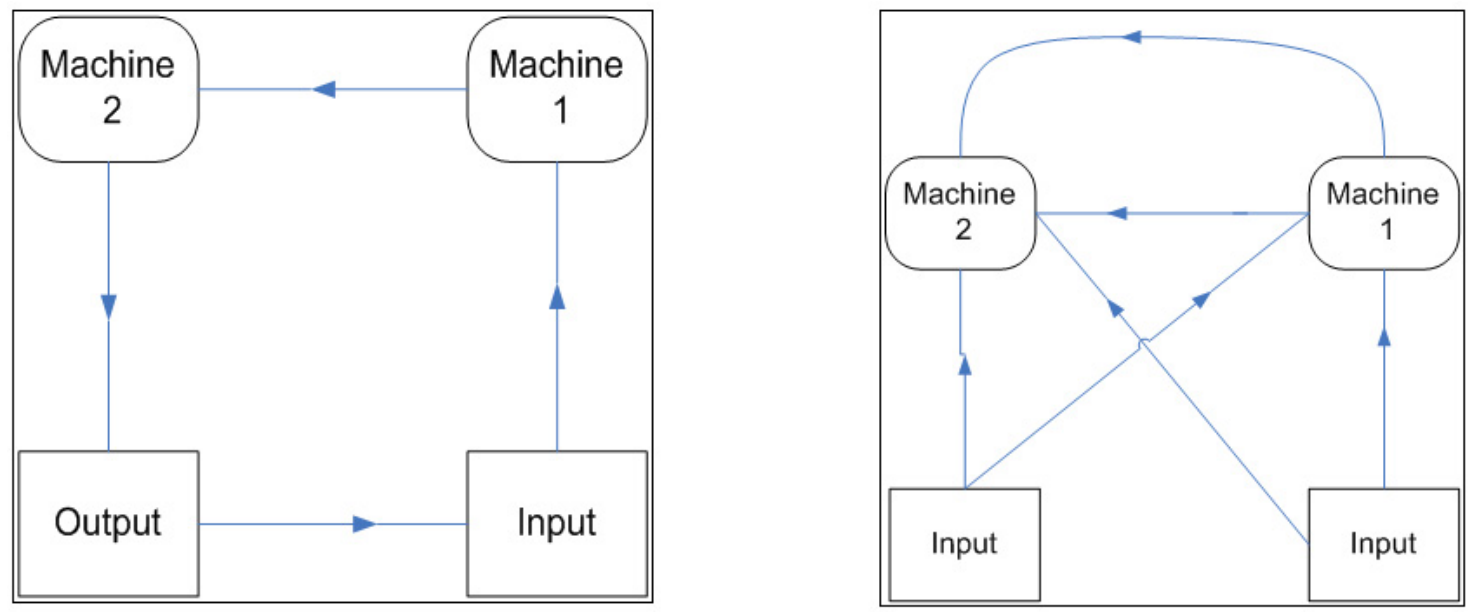

Fig. 4. Robot move cycles $S_{1}$ and $S_{2}$ in robotic cell with two machines 


\subsection{Fuzzy cycle time in three-machine-multiple-part type robotic cells}

Sethi et al. ${ }^{[25]}$ prove that there is exactly $\mathrm{m}$ ! Potentially schedules for one-part type cycles in an machine cells. So, for a three machine robotic cell, there are six potentially schedules $\left(S_{1}, S_{2}, S_{3}, S_{4}, S_{5}, S_{6}\right)$ for $\mathrm{MRC}_{3} \mid k=1,1-$ unit $\mid C_{t}$, respectively .In this research, we use fuzzy numbers for calculating the related cycle times. The fuzzy cycle times of different schedules are as follows:

For cycle $S_{1}$ :

$$
\begin{aligned}
& \tilde{T}_{1(\sigma)}=n \tilde{\alpha}_{1}+\sum_{i=1}^{n}\left(\tilde{\theta}_{i}+\tilde{\lambda}_{i}+\tilde{\varphi}_{i}\right) \\
& \tilde{\alpha}_{1}=4 \tilde{\delta}_{0}+2 \sum_{i=1}^{4} \tilde{\delta}_{i}+\sum_{i=1}^{8} \tilde{\varepsilon}_{i}-3 \tilde{\gamma}
\end{aligned}
$$

For cycle $S_{2}$ :

$$
\begin{aligned}
& \tilde{T}_{2(\sigma)}=n \tilde{\alpha}_{2}+\sum_{i=1}^{n} \operatorname{Max}\left\{\tilde{\beta}_{2}, \tilde{\alpha}_{2 \sigma(i+2)}, \tilde{\lambda}_{2 \sigma(i+1)}-\tilde{W}_{i}^{3}\right\}+\sum_{i=1}^{n} \tilde{W}_{i}^{3} \\
& \tilde{\beta}_{2}=\sum_{i=1}^{8} \tilde{\varepsilon}_{i}+4 \tilde{\delta}_{0}+2\left(\tilde{\delta_{1}}+\tilde{\delta_{2}}+\tilde{\delta_{3}}+\tilde{\delta}_{4}\right)-3 \tilde{\gamma} \\
& \tilde{\alpha}_{2}=4 \tilde{\delta}_{0}+2\left(\tilde{\delta}_{2}+\tilde{\delta_{3}}\right)-\tilde{\gamma} \\
& \tilde{W}_{3}^{i}=\operatorname{Max}\left\{\tilde{\beta}_{2}, \tilde{\theta}_{2 \sigma(i+1)}-\max \left\{\tilde{0}, \tilde{\lambda}_{2 \sigma(i)}-\tilde{\beta}_{2}-\tilde{W}_{3}^{i-1}\right\}\right\} \quad i=1,2, \cdots, n \\
& \tilde{\lambda}_{2 \sigma(i)}=\tilde{\lambda}_{\sigma(i)}+\sum_{i=3}^{6} \tilde{\varepsilon_{i}} \quad i=1,2, \cdots, n \\
& \tilde{\theta}_{2 \sigma(i)}=\tilde{\theta}_{\sigma(i)}+\sum_{i=1}^{4} \tilde{\varepsilon_{i}}+2 \tilde{\delta}_{1}+22 \tilde{\delta_{4}}+\tilde{\varepsilon_{7}}+\tilde{\varepsilon_{8}}-\tilde{\gamma} \quad i=1,2, \cdots, n \\
& \tilde{\varphi}_{2 \sigma(i)}=\tilde{\varphi}_{\sigma(i)}+\sum_{i=5}^{8} \tilde{\varepsilon_{i}}+2 \tilde{\delta_{1}}+22 \tilde{\delta}_{4}+\tilde{\varepsilon_{1}}+\tilde{\varepsilon_{2}}-2 \tilde{\gamma} \quad i=1,2, \cdots, n
\end{aligned}
$$

For cycle $S_{3}$ :

$$
\begin{aligned}
& \tilde{T}_{3(\sigma)}=n \tilde{\alpha}_{3}+\sum_{i=1}^{n} \operatorname{Max}\left\{\tilde{e}_{3 \sigma(i+1)}, \tilde{\varphi}_{3 \sigma(i)}\right\} \\
& \tilde{\lambda}_{3 \sigma(i)}=\tilde{\lambda}_{\sigma(i)}+2\left(\tilde{\delta}_{0}+\tilde{\delta}_{1}+\tilde{\delta}_{2}\right)+\sum_{i=1}^{4} \tilde{\varepsilon}_{i}-\tilde{\gamma} \quad i=1,2, \cdots, n \\
& \tilde{\varphi}_{3 \sigma(i)}=\tilde{\varphi}_{\sigma(i)}+2 \tilde{\delta}_{4}+\tilde{\varepsilon}_{7}+\tilde{\varepsilon}_{8} \quad i=1,2, \cdots, n \\
& \tilde{e}_{3 \sigma(i)}=\operatorname{Max}\left\{\tilde{\beta}_{3}+\tilde{\alpha}_{\sigma(i)}, \tilde{\lambda}_{3 \sigma(i)}+\tilde{\varphi}_{\sigma(i)}\right\} \quad \tilde{\alpha}_{3}=2 \tilde{\delta}_{0}+2 \tilde{\delta}_{3}+\tilde{\varepsilon}_{5}+\tilde{\varepsilon}_{6}-\tilde{\gamma}
\end{aligned}
$$

For cycle $S_{4}$ :

$$
\begin{aligned}
& \tilde{T}_{4(\sigma)}=n \tilde{\alpha}_{4}+\sum_{i=1}^{n} \tilde{\lambda}_{\sigma(i)}+\sum_{i=1}^{n} \operatorname{Max}\left\{\tilde{\beta}_{4}, \tilde{\theta}_{4 \sigma(i+1)}, \tilde{\varphi}_{4 \sigma(i)}\right\} \\
& \tilde{\alpha}_{4}=4 \tilde{\delta}_{0}+2\left(\tilde{\delta}_{2}+\tilde{\delta}_{3}\right)+\sum_{i=3}^{6} \tilde{\varepsilon}_{i}-2 \tilde{\gamma} \\
& \tilde{\beta}_{4}=\sum_{i=1}^{2} \tilde{\varepsilon}_{i}+\sum_{i=7}^{8}+4 \tilde{\delta}_{0}+2\left(\tilde{\delta}_{1}+\tilde{\delta}_{2}+\tilde{\delta}_{3}+\tilde{\delta}_{4}\right)-3 \tilde{\gamma} \\
& \tilde{\varphi}_{4 \sigma(i)}=\tilde{\varphi}_{\sigma(i)}+2 \tilde{\delta}_{4}+\tilde{\varepsilon}_{7}+\tilde{\varepsilon}_{8} \quad i=1,2, \cdots, n \\
& \tilde{\theta}_{4 \sigma(i)}=\tilde{\theta}_{\sigma(i)}+2 \tilde{\delta}_{1}+\tilde{\varepsilon}_{1}+\tilde{\varepsilon}_{2} \quad i=1,2, \cdots, n
\end{aligned}
$$

For cycle $S_{5}$ :

$$
\begin{aligned}
& \tilde{T}_{5(\sigma)}=n \tilde{\alpha}_{5}+\sum_{i=1}^{n} \operatorname{Max}\left\{\tilde{\theta}_{5 \sigma(i+1)}, \tilde{f}_{5 \sigma(i)}\right\} \\
& \tilde{\theta}_{5 \sigma(i)}=\tilde{\theta}_{\sigma(i)}+2 \tilde{\delta}_{1}+\tilde{\varepsilon}_{1}+\tilde{\varepsilon}_{2} \quad i=1,2, \cdots, n \\
& \tilde{\lambda}_{5 \sigma(i)}=\tilde{\lambda}_{\sigma(i)}+\sum_{i=5}^{8}+2 \tilde{\delta}_{0}+2 \tilde{\delta}_{3}+2 \tilde{\delta}_{4}+\tilde{\varepsilon}_{1}+\tilde{\varepsilon}_{2}-\tilde{\gamma} \quad i=1,2, \cdots, n \\
& \tilde{\beta}_{5}=4 \tilde{\delta}_{0}+2\left(\tilde{\delta}_{1}+\tilde{\delta}_{2}+\tilde{\delta}_{3}+\tilde{\delta}_{4}\right) \tilde{\varepsilon}_{1}+\tilde{\varepsilon}_{2}+\sum_{i=5}^{8} \tilde{\varepsilon}_{i}-2 \tilde{\gamma} \\
& \tilde{f}_{5 \sigma(i)}=\operatorname{Max}\left\{\tilde{\beta}_{5}+\tilde{\theta}_{\sigma(i)}, \tilde{\lambda}_{5 \sigma(i)}+\tilde{\varphi}_{\sigma(i)}\right\} \quad \tilde{\alpha}_{5}=2 \tilde{\delta}_{0}+2 \tilde{\delta}_{2}+\tilde{\varepsilon}_{3}+\tilde{\varepsilon}_{4}-\tilde{\gamma}
\end{aligned}
$$


For cycle $S_{6}$ :

$\tilde{T}_{6(\sigma)}=n \tilde{\alpha}_{6}+\sum_{i=1}^{n} \operatorname{Max}\left\{\tilde{\beta}_{6}, \tilde{\theta}_{6 \sigma(i)}-\tilde{W}_{1}^{i+1}, \tilde{\lambda}_{6 \sigma(i+1)}, \tilde{\theta}_{6 \sigma(i+2)}\right\}$

$\tilde{W}_{1}^{i+1}=\operatorname{Max}\left\{\tilde{0}, \tilde{\theta}_{6 \sigma(i)}-\operatorname{Max}\left\{\tilde{\beta}_{6}, \tilde{\lambda}_{6 \sigma(i)}, \tilde{\varphi}_{6 \sigma(i-1)}-\tilde{W}_{1}^{i}\right\}\right\} \quad i=1,2, \cdots, n$

$\tilde{\lambda}_{6 \sigma(i)}=\tilde{\lambda}_{\sigma(i)}+\sum_{i=3}^{6} \tilde{\varepsilon}_{i}+2 \tilde{\delta}_{0}+2 \tilde{\delta}_{2}+2 \tilde{\delta}_{3}-\tilde{\gamma} \quad i=1,2, \cdots, n$

$\tilde{\theta}_{6 \sigma(i)}=\tilde{\theta}_{\sigma(i)}+\sum_{i=1}^{4} \tilde{\varepsilon}_{i}+2 \tilde{\delta}_{1}+2 \tilde{\delta}_{2}-\tilde{\gamma} \quad i=1,2, \cdots, n$

$\tilde{\varphi}_{6 \sigma(i)}=\tilde{\varphi}_{\sigma(i)}+\sum_{i=8}^{5} \tilde{\varepsilon}_{i}+2 \tilde{\delta}_{3}+2 \tilde{\delta}_{4}-\tilde{\gamma} \quad i=1,2, \cdots, n$

$\tilde{\beta}_{6}=\sum_{i=1}^{8} \tilde{\varepsilon}_{i}+6 \tilde{\delta}_{0}+2\left(\tilde{\delta}_{1}+2 \tilde{\delta}_{2}+2 \tilde{\delta}_{3}+\tilde{\delta}_{4}\right)-4 \tilde{\gamma}$

$\tilde{\alpha}_{6}=2 \tilde{\delta}_{0}$

Hall et al. ${ }^{[9]}$ show that the part sequencing problem under robot move cycles $S_{3}, S_{4}, S_{5}$ can be reduced to a problem of the form : minimize $\left(\tilde{C}+\sum \max \left(\tilde{e}_{\sigma(i+I)}\right), \tilde{f}_{\sigma(i)}\right)$ for some constant $\mathrm{C}$. Any such problem can be solved optimally in $\mathrm{O}(\mathrm{nlog}(\mathrm{n}))$ time by Gilmore and Gomory algorithm ${ }^{[7]}$. We recall that, since the above times are fuzzy, we should use fuzzy Gilmore and Gomory algorithm.

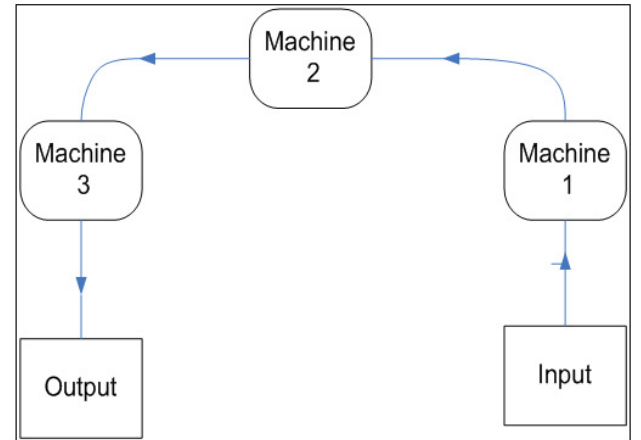

Fig. 5. Cycle $S_{1}$ for three-machine robotic cell

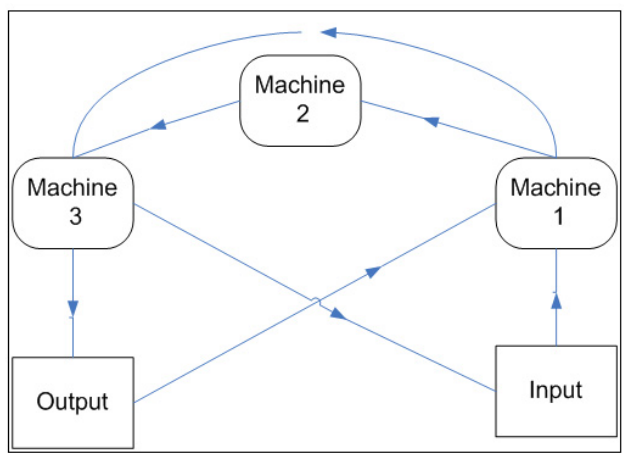

Fig. 7. Cycle $S_{3}$ for three-machine robotic cell

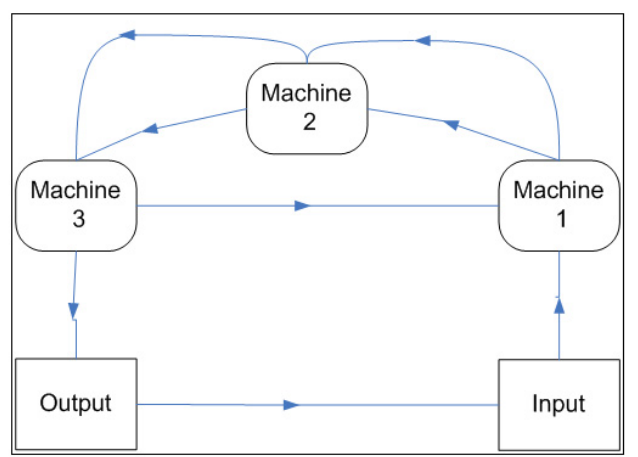

Fig. 6. Cycle $S_{2}$ for three-machine robotic cell

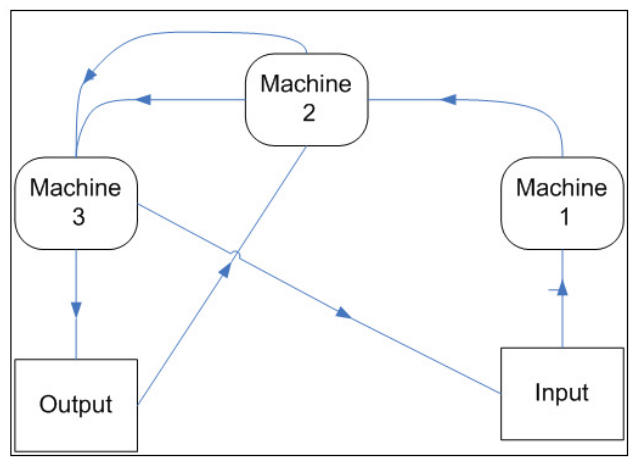

Fig. 8. Cycle $S_{4}$ for three-machine robotic cell

\section{Determination of fuzzy cycle times for $s_{2}$ in two machine and $s_{3}, s_{4}$ and $s_{5}$ in three-machine robotic cells}

In this paper, we deal with three robot move cycles $\left(S_{3}, S_{4}, S_{5}\right)$ in Mobile robotic cell with three machines $\left(\mathrm{MRC}_{3}\right)$, and $S_{2}$ in Mobile robotic cell with two machines $\left(\mathrm{MRC}_{2}\right)$ that produce multiple part type. All these 


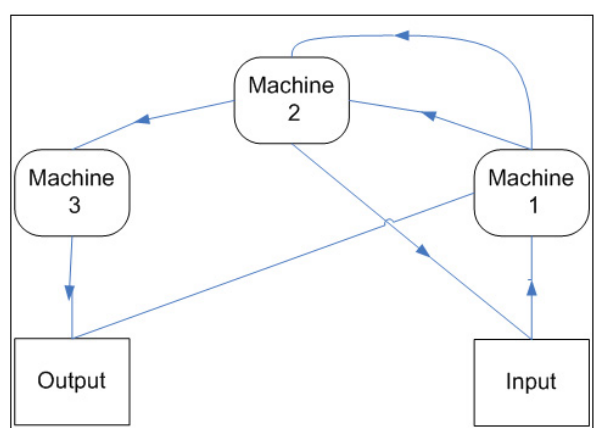

Fig. 9. Cycle $S_{5}$ for three-machine robotic cell

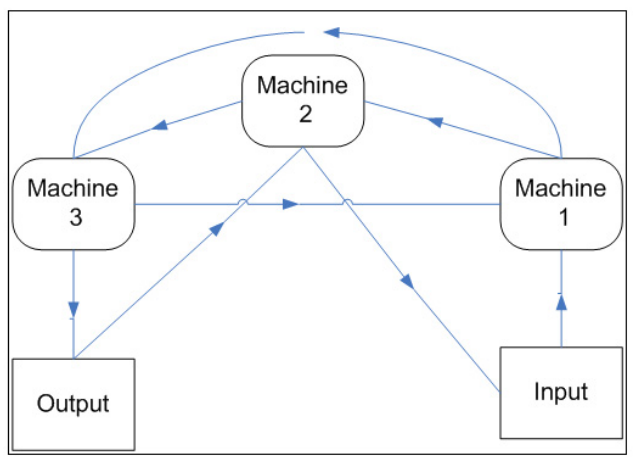

Fig. 10. Cycle $S_{6}$ for three-machine robotic cell

problems can be solved in polynomial time with Gilmore and Gomory algorithm ${ }^{[7]}$. First, we fuzzify the problem of minimization of cycle time $\left(C_{t}\right)$ in $\mathrm{MRC}_{2}$ and $\mathrm{MRC}_{3}$ and, then we expand the Gilmore and Gomory algorithm to fuzzy Gilmore and Gomory algorithm. To fuzzify the robotic cell problems, as stated in section 4, we suppose that the processing time of all jobs on machines, the robot travel times, and the times of picking up, loading, unloading and dropping out of parts are all fuzzy numbers. It should be noted that in two-machine robotic cells $S_{1}$ is very easy and $S_{2}$ can be solved by Gilmore and Gomory algorithm and in three-machine robotic cells $S_{1}$ is easy and $S_{2}$ and $S_{6}$ are NP-hard and $S_{3}, S_{4}, S_{5}$ can be solved by Gilmore and Gomory algorithm.

It should be noted that in this paper, for the aim of simplicity, the Triangular (T. F. N.) and the Trapezoidal (Tr. F. N.) membership functions are used for fuzzy numbers (Fig. 11 and 12).

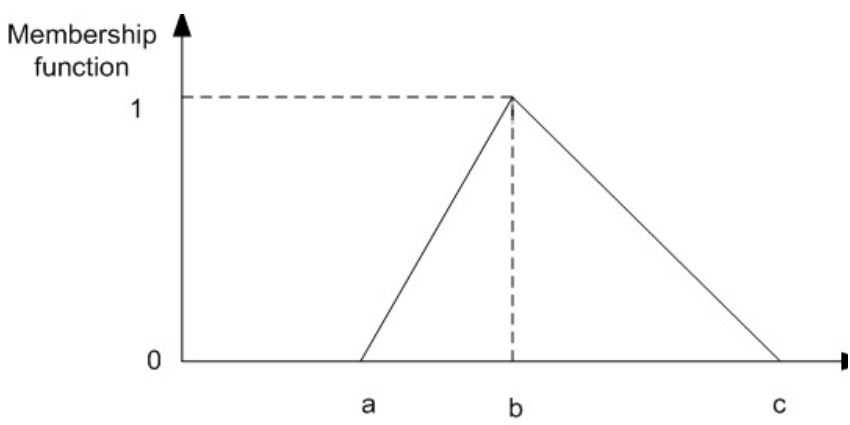

Fig. 11. Triangular fuzzy number

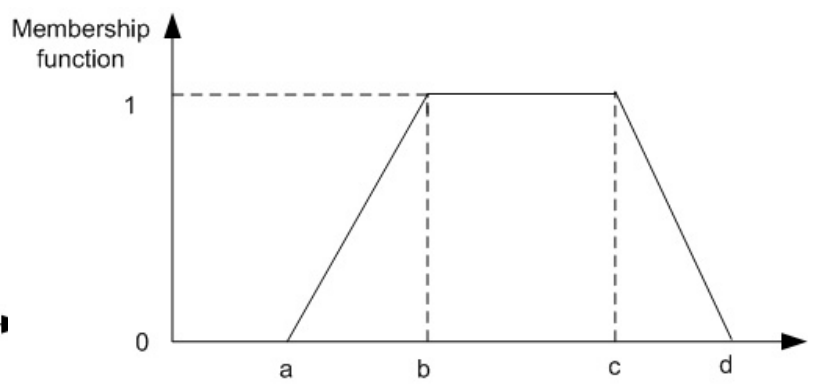

Fig. 12. Trapozeidal fuzzy number

To calculate the fuzzy cycle times and fuzzy waiting times of robot on machines, we used $\alpha$-cut of fuzzy sets for maximization, minimization, addition and subtraction operations. Since $\alpha$-cut operates on interval values, we first convert the Triangular and Trapezoidal membership functions to $\alpha$-cut intervals, $\alpha \in[0,1]$, and, then, we use the operators. Transformation of the ordinary fuzzy numbers into $\alpha$-cut format for T. F. N and Tr. F. N are shown in Fig. 13 and 14.

$\mu(x)=\left\{\begin{array}{cc|}0 & \text { if } x<a \\ \frac{x-a}{b-a} & \text { if } a \leq x \leq b \\ \frac{c-x}{c-b} & \text { if } b<x \leq c \\ 0 & \text { if } x>c\end{array} \Rightarrow A_{\alpha}=[(b-a) \cdot \alpha+a,(b-c) \cdot \alpha+c]\right.$

Fig. 13. Transformation of triangular membership function into $\alpha$-cut 


$$
\mu(x)=\left\{\begin{array}{cl}
0 & \text { if } x<a, x>d \\
\frac{x-a}{b-a} & \text { if } a \leq x \leq b \\
1 & \text { if } b<x \leq c \\
\frac{d-x}{d-c} & \text { if } c<x \leq d
\end{array} \Rightarrow A_{\alpha}=[(b-a) \cdot \alpha+a,(b-c) \cdot \alpha+c]\right.
$$

Fig. 14. Transformation of trapezoidal membership function into $\alpha$-cut

Using $\alpha$-cut format, the main operators used in this paper are as follows:

a. Maximum operator:

$$
\operatorname{Max}\left(\left[a_{1}, b_{1}\right],\left[a_{2}, b_{2}\right]\right)=\left[\operatorname{Max}\left(a_{1}, b_{1}\right), \operatorname{Max}\left(a_{2}, b_{2}\right)\right]
$$

b. Minimum operator:

$$
\operatorname{Min}\left(\left[a_{1}, b_{1}\right],\left[a_{2}, b_{2}\right]\right)=\left[\operatorname{Min}\left(a_{1}, b_{1}\right), \operatorname{Min}\left(a_{2}, b_{2}\right)\right]
$$

c. Addition operator:

$$
\left[a_{1}, b_{1}\right]+\left[a_{2}, b_{2}\right]=\left[a_{1}+a_{2}, b_{1}+b_{2}\right]
$$

d. Subtraction:

$$
\left[a_{1}, b_{1}\right]-\left[a_{2}, b_{2}\right]=\left[a_{1}-b_{2}, b_{1}-a_{2}\right]
$$

e. Image:

$$
-\left[a_{1}, b_{1}\right]=\left[-b_{1},-a_{1}\right]
$$

The defuzzification method used in this paper is "BADD" method proposed by Yager ${ }^{[28]}$. In this formulation, " $h$ " is the interval $[0,1]$ that is divided into $\mathrm{n}$ parts and " $\tilde{A}$ " is the fuzzy number converted to $\alpha$ - cut number with " $h$ " and $\beta$ is the defuzzification parameter.

$$
\begin{gathered}
h=[0, \Delta \alpha, 2 \Delta \alpha, \cdot 1]_{1 \times n} ; \quad \tilde{A}=\left[\begin{array}{cc}
l l a_{0} & b_{0} \\
a_{1} & b_{1} \\
\cdot & \cdot \\
\cdot & \cdot \\
\cdot & \cdot \\
a_{n} & b_{n}
\end{array}\right]_{(n \times 2)} \\
\operatorname{Defuzzify}(\tilde{A})=\frac{\sum_{i=1}^{n}\left(h_{i}\right)^{\beta} \cdot A_{i 1}+\sum_{i=1}^{n}\left(h_{i}\right)^{\beta} \cdot \tilde{A_{i 2}}}{2 \cdot \sum_{i=1}^{n}\left(h_{i}\right)^{\beta}}
\end{gathered}
$$

In (5), if $\beta \rightarrow 1$ then equation (5) moves to Center of Area (COA):

$$
H(\tilde{A})=\frac{\sum_{i=1}^{n} h \cdot \tilde{A}}{2 \cdot \sum_{i=1}^{n} h_{i}}
$$




\section{Fuzzy gilmore and gomory algorithm}

As discussed in section 2, cycle time in the problems $M R C_{2}\left|k>2, S_{2}\right| C_{t}$ and $M R C_{3} \mid k>$ $2, S_{3}, S_{4}, S_{5} \mid C_{t}$ can be written as $\tilde{C}_{t}=C+\sum \operatorname{Max}\left(e_{(i+1)}, \tilde{f_{(i)}}\right)$ where, $C$ is a constant and $\sigma$ is the sequence of parts. Any problem which seems to have such a form can be solved by Gilmore and Gomory algorithm. As such, we fuzzify this algorithm to obtain optimal fuzzy cycle time for the above problems and the sequence of parts which Leeds to it. For this purpose, we assume the followings:

- The ready time of all jobs is fuzzy zeros: $\tilde{0}$

- The processing time of all jobs on each machine is fuzzy Triangular or Trapezoidal fuzzy Number.

- $\tilde{e}_{i}$ is fuzzy processing time of $i$ th job on Machine 1.

- $\tilde{f}_{i}$ is fuzzy processing time of $i$ th job on Machine 2 .

- $C_{i, i+1}$ is fuzzy cost function for connecting node $i$ to node $i+1$.

- $\psi$ is minimal tour that leads to optimal cycle time.

Here, for sorting the fuzzy number, the following strategies are adopted:

i. For Triangular Fuzzy Numbers, the mean method is used. Let A and B be two Triangular fuzzy numbers: $A=\left(a_{A}, b_{A}, c_{A}\right) ; B=\left(a_{B}, b_{B}, c_{B}\right)$ and $H$ be function as follows:

$$
H(A)=\frac{1}{3} \times\left(a_{A}+b_{A}+c_{A}\right) ; H(B)=\frac{1}{3} \times\left(a_{B}+b_{B}+c_{B}\right)
$$

Using this method, we say $A>B$ if $H(A)>H(B)$.

ii. For Trapezoidal Fuzzy Numbers, a ranking method obtained from center of area defuzzification method is generated. Let $A$ and $B$ be two Trapezoidal fuzzy numbers : $A=\left(a_{A}, b_{A}, c_{A}, d_{A}\right), B=$ $\left(a_{B}, b_{B}, c_{B}, d_{B}\right)$ and let " $H$ " be:

$$
H=\frac{\int x \mu(x) d(x)}{\int \mu(x) d(x)}
$$

Then

$$
H(A)=\frac{1}{3} \times\left(a_{A}+b_{A}+c_{A}+d_{A}\right)-\frac{1}{3} \times\left(\frac{c_{A} d_{A}-a_{A} b_{A}}{d_{A}-a_{A}+c_{A}-b_{A}}\right)
$$

And

$$
H(B)=\frac{1}{3} \times\left(a_{B}+b_{B}+c_{B}+d_{B}\right)-\frac{1}{3} \times\left(\frac{c_{B} d_{B}-a_{B} b_{B}}{d_{B}-a_{B}+c_{B}-b_{B}}\right)
$$

Using this method, we say $A>B$ if $H(A)>H(B)$.

iii. For transforming fuzzy numbers to their related -cuts, we use the following formula:

$$
h=[0, \Delta \alpha, 2 \Delta \alpha, \cdots, 1]_{1 \times n} ; \tilde{A}=\left[\begin{array}{cc}
l l a_{0} & b_{0} \\
a_{1} & b_{1} \\
\cdot & \cdot \\
\cdot & \cdot \\
\cdot & \cdot \\
a_{n} & b_{n}
\end{array}\right]_{(n \times 2)} ; H(\tilde{A})=\frac{\sum_{i=1}^{n} h \cdot \tilde{A}}{2 \cdot \sum_{i=1}^{n} h_{i}}
$$

The steps of the proposed fuzzy Gilmore and Gomory algorithm are as follows:

Step 1. Read two series of fuzzy processing times:

$\tilde{e}_{i}$ : fuzzy process time on machine 1 ;

$\tilde{f}_{i}$ : fuzzy process time on machine 2 ; if the fuzzy process time is Triangular, for $i=1,2, \cdots, n$, then entered it as $\tilde{x}_{i}=\left(a_{i}, b_{i}, c_{i}\right)$; if the fuzzy processing time is Trapezoidal, then entered it as $\tilde{x}=\left(a_{i}, b_{i}, c_{i}, d_{i}\right)$.

Step 2. Rank the fuzzy numbers according to the kind of their membership function:

1) For triangular numbers $H\left(\tilde{x}_{i}\right)=\frac{1}{3}\left(a_{i}+b_{i}+c_{i}\right)$.

2) For trapezoidal Fuzzy numbers $H\left(\tilde{x}_{i}\right)=\frac{1}{3}\left(a_{i}+b_{i}+c_{i}+d_{i}\right)-\frac{1}{3}\left(\frac{c_{i} d_{i}-a_{i} b_{i}}{d_{i}-a_{i}+c_{i}-b_{i}}\right)$.

Step 3. Arrange the numbers $H\left(\tilde{f}_{i}\right)$ and renumber the jobs so that write the new numbering $H\left(\tilde{f}_{i}\right)<H\left(\tilde{f_{i+1}}\right)$ $i=1,2,3, \cdots, n-1$. 
Step 4. Arrange the $H\left(\tilde{e}_{i}\right)$ in no decreasing order of its size and find the $\varphi(p)$ for all $p$. The permutation $\varphi$ is defined by $\varphi(p)=q, q$ being such that $H\left(e_{i}\right)$ is the $p$-th smallest of all $H\left(e_{i}\right)$ 's.

Step 5. Compute the fuzzy cost with fuzzy operations:

$$
\tilde{C}_{i, j+1}=\max \left\{\tilde{0},\left\{\min \left(\tilde{f}_{i+1}, \tilde{e}_{\phi(i+1)}\right)-\max \left(\tilde{f}, \tilde{e}_{\phi(i)}\right)\right\}\right\}, \text { for } i=1,2,3, \cdots, n-1
$$

Step 6. Construct a fuzzy undirected graph with n nodes and undirected $\operatorname{arcs}(i, \varphi(i)) ; i=1,2, \cdots, n$.

Step 7. If the current fuzzy graph has only one component, go to Step 9; otherwise select the smallest value $H\left(\tilde{C}_{i, i+1} 1\right)$ such that $i$ is in one component and $i+1$ in another. In the case of tie for smallest, choose any.

Step 8. Adjoin the undirected arc $(i, i+1)$ to the fuzzy graph using the i value selected in Step 7.

Step 9. Divide the arcs added in step 8 into two groups: i) arcs for which $H\left(\tilde{e}_{\phi(i)}\right) \geq H\left(\tilde{f}_{i}\right)$ in group 1. ii) acrs for which $H\left(\tilde{e}_{\phi}(i)\right) \geq H\left(\tilde{f}_{i}\right)$ in group 2 .

Step 10. Find the largest index $i_{1}$ such that $\operatorname{arc}\left(i_{1}, i_{1+1}\right)$ is in group 1 . Find the second largest $i_{2}$, etc, up to $i_{r}$, assuming there are "r" elements in group 1.

Step 11. find the smallest index $j_{1}$ such that arc $\left(j_{1}, j_{1+1}\right)$ is in group 2. Find the second largest $j_{2}$, etc. up to $j_{k}$, assuming there are " $\mathrm{k}$ " elements in group 2 .

Step 12. the minimal cycle time is obtained by following the $i^{\text {th }}$ part by the part $\psi(i)$, where

$$
\psi(i)=\varphi_{\alpha_{i_{1}, i_{1}+1} \alpha_{i_{2}, i_{2}+1} \cdots \alpha_{i_{r}, i_{r}+1} \alpha_{j_{1}, j_{1}+1} \alpha_{j_{2}, j_{2}+1} \cdots \alpha_{j_{k}, j_{k}+1}}
$$

In the above the permutation $\alpha_{p, q}$ is defined to be: $\alpha_{p, q}(p)=q ; \alpha_{p, q}(q)=p ; \alpha_{p, q}(i)=i \quad$ if $i \neq p, q$.

Step 13. Renumber the part as they are before Step 3.

\section{Numerical examples}

This section presents two numerical examples of $M R C_{3}\left|K \geq 2, S_{4}\right| C_{t}$ and $M R C_{3}\left|K \geq 2, S_{3}\right| C_{t}$ and compares the result of their related crisp and fuzzy cycle times. In any case, each problem contains 10 jobs.

$M R C_{3}\left|K \geq 2, S_{4}\right| C_{t}$

Crisp problem: Tab. 1 shows the processing times of 10 jobs (parts) in a robotic cell with three machines:

Table 1. The processing times of 10 jobs in a robotic cell with three machines

\begin{tabular}{c|c|c|c|c|c|c|c|c|c|c|c}
\hline \multicolumn{10}{c}{ Job } \\
\hline & & 1 & 2 & 3 & 4 & 5 & 6 & 7 & 8 & 9 & 10 \\
\hline \multirow{3}{*}{ Process Time } & $\theta_{i}$ & 19 & 10 & 9 & 22 & 26 & 14 & 20 & 26 & 12 & 15 \\
\cline { 2 - 13 } & $\lambda_{i}$ & 8 & 6 & 12 & 9 & 4 & 2 & 5 & 4.5 & 11 & 8 \\
\cline { 2 - 12 } & $\varphi_{i}$ & 27 & 29 & 51 & 65 & 11 & 25 & 49 & 37 & 19 & 9.5 \\
\hline
\end{tabular}

Moreover, the pick up, dropping on and travel times of robot are demonstrated in Tab. 2:

Table 2. The processing times of 10 jobs in a robotic cell with three machines

\begin{tabular}{c|c|c|c|c|c|c|c}
\hline$\varepsilon_{1}=0.5$ & $\varepsilon_{2}=0.6$ & $\varepsilon_{3}=1.5$ & $\varepsilon_{4}=2.2$ & $\varepsilon_{5}=0.8$ & $\varepsilon_{6}=1$ & $\varepsilon_{7}=1.6$ & $\varepsilon_{8}=0.4$ \\
\hline$\delta_{0}=1.5$ & $\delta_{1}=3$ & $\delta_{2}=2$ & $\delta_{3}=1$ & $\delta_{4}=1.4$ & & & \\
\hline$\gamma=0.5$ & \multicolumn{10}{|c}{} \\
\hline
\end{tabular}

Using Gilmore and Gomory algorithm that was encoded in MATLAB software, the optimal sequence of jobs and its related cycle time are as follows:

\begin{tabular}{|c|c|}
\hline Optimal Sequence & Cycle time \\
\hline$Q^{*}=10,9,6,2,7,4,8,1,3,5$ & $C_{t}=619.7$ \\
\hline
\end{tabular}




\section{Fuzzy problem:}

Now, the above crisp problem is fuzzufied. In other words, the processing times of jobs on machines, pick up, drop on and robot moves are trapezoidal fuzzy numbers with parameters $\left[a_{i}, b_{i}, c_{i}, d_{i},\right]$. The processing times of parts on $M_{1}, M_{2}$, and $M_{3}$ are shown in Tab. 3, 4, and 5.

Table 3. Fuzzy processing times of jobs on machine 1.

\begin{tabular}{|c|c|c|c|c|c|c|c|c|c|c|c|}
\hline \multicolumn{12}{|c|}{ Job } \\
\hline & & 1 & 2 & 3 & 4 & 5 & 6 & 7 & 8 & 9 & 10 \\
\hline \multirow{4}{*}{ Parameter } & $a_{i}$ & 18 & 9.6 & 8 & 21 & 25 & 13.6 & 19.2 & 24.4 & 11 & 14 \\
\hline & $b_{i}$ & 18.8 & 9.8 & 8.8 & 21.8 & 25.8 & 13.8 & 19.8 & 25.8 & 11.8 & 14.8 \\
\hline & $c_{i}$ & 19.2 & 10.2 & 9.2 & 22.2 & 26.2 & 14.2 & 20.2 & 26.2 & 12.2 & 15.2 \\
\hline & $d_{i}$ & 20 & 10.8 & 10 & 23 & 27 & 14.4 & 21 & 26.8 & 13.6 & 16.2 \\
\hline
\end{tabular}

Table 4. Fuzzy processing times of jobs on machine 2 .

\begin{tabular}{c|c|c|c|c|c|c|c|c|c|c|c}
\hline \multicolumn{1}{c}{} & \multicolumn{10}{c}{ Job } \\
\hline & & 1 & 2 & 3 & 4 & 5 & 6 & 7 & 8 & 9 & 10 \\
\hline \multirow{4}{*}{ Parameter } & $a_{i}$ & 7.3 & 5.5 & 11.4 & 8 & 3.6 & 1.4 & 4.6 & 4 & 10.3 & 7.4 \\
\cline { 2 - 12 } & $b_{i}$ & 7.8 & 5.8 & 11.8 & 8.8 & 3.8 & 1.8 & 4.8 & 4.3 & 10.8 & 7.8 \\
\cline { 2 - 12 } & $c_{i}$ & 8.2 & 6.2 & 12.2 & 9.2 & 4.2 & 2.2 & 5.2 & 4.7 & 11.2 & 8.2 \\
\cline { 2 - 11 } & $d_{i}$ & 8.5 & 6.5 & 12.6 & 10 & 4.4 & 2.9 & 5.4 & 5 & 11.5 & 8.5 \\
\hline
\end{tabular}

Table 5. Fuzzy processing times of jobs on machine 3 .

\begin{tabular}{c|c|c|c|c|c|c|c|c|c|c|c}
\hline \multicolumn{10}{c}{ Job } \\
\hline & & 1 & 2 & 3 & 4 & 5 & 6 & 7 & 8 & 9 & 10 \\
\hline \multirow{3}{*}{ Parameter } & $a_{i}$ & 22.2 & 27.8 & 46.6 & 59 & 9 & 21.8 & 45 & 34.2 & 13.4 & 7.4 \\
\cline { 2 - 14 } & $b_{i}$ & 26.8 & 28.8 & 50.8 & 64.8 & 10.8 & 24.8 & 48.8 & 36.8 & 16.8 & 9.3 \\
\cline { 2 - 13 } & $c_{i}$ & 27.2 & 29.2 & 51.2 & 65.2 & 11.2 & 25.2 & 49.2 & 37.2 & 17.2 & 9.7 \\
\cline { 2 - 12 } & $d_{i}$ & 31 & 32.6 & 55.4 & 71 & 13 & 27.8 & 53 & 42.2 & 17.8 & 11.8 \\
\hline
\end{tabular}

The Fuzzy pick up, drop on and robot move time are shown in Tab. 6 and 7.

Table 6. Fuzzy pick up, drop on times of robot.

\begin{tabular}{c|c|c|c|c|c|c|c|c|c}
\hline \multicolumn{1}{c}{ Job } \\
\hline & & $\varepsilon_{1}$ & $\varepsilon_{2}$ & $\varepsilon_{3}$ & $\varepsilon_{4}$ & $\varepsilon_{5}$ & $\varepsilon_{6}$ & $\varepsilon_{7}$ & $\varepsilon_{8}$ \\
\hline \multirow{4}{*}{ Parameter } & $a_{i}$ & 0.4 & 0.5 & 1 & 2.1 & 0.7 & 0.9 & 1.5 & 0.2 \\
\cline { 2 - 11 } & $b_{i}$ & 0.45 & 0.55 & 1.45 & 2.15 & 0.75 & 0.95 & 1.55 & 0.35 \\
\cline { 2 - 11 } & $c_{i}$ & 0.55 & 0.65 & 1.55 & 2.25 & 0.85 & 1.05 & 1.65 & 0.45 \\
\cline { 2 - 10 } & $d_{i}$ & 0.6 & 0.7 & 2 & 2.3 & 0.9 & 1.1 & 1.8 & 0.6 \\
\hline
\end{tabular}

Using the developed fuzzy Gilmore and Gomory algorithm, the optimal schedule is: $Q^{*}=\{10,9,3,8$ $, 7,4,5,2,1,6\}$

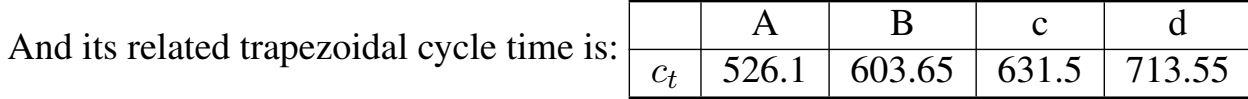

Fig. 15 demonstrates fuzzy cycle time of $S_{4}$. Using BADD defuzzification method with $\beta=100$, leads to defuzzified cycle time $D F\left(C_{t}\right)=616.38$, which is rather smaller than it's related crisp cycle time.

B. $M R C_{3}\left|k \geq 2, S_{3}\right| C_{t}$

Crisp problem: 
Table 7. Fuzzy travel times of robot.

\begin{tabular}{c|c|c|c|c|c|c|c|c}
\hline \multicolumn{2}{c}{} & \multicolumn{8}{c}{ Job } \\
\hline \multirow{4}{*}{ Parameter } & & $\delta_{0}$ & $\delta_{1}$ & $\delta_{2}$ & $\delta_{3}$ & $\delta_{4}$ & $\Gamma$ & Z(Fuzzy Zero) \\
\cline { 2 - 9 } & $a_{i}$ & 1 & 2.5 & 1.8 & 0.8 & 1.1 & 0.45 & -0.5 \\
\cline { 2 - 9 } & $b_{i}$ & 1.45 & 2.95 & 1.95 & 0.95 & 1.35 & 0.5 & -0.25 \\
\cline { 2 - 9 } & $c_{i}$ & 1.55 & 3.05 & 2.05 & 1.05 & 1.45 & 0.55 & 0.25 \\
\cline { 2 - 9 } & $d_{i}$ & 2 & 3.5 & 2.2 & 1.2 & 1.7 & 0.6 & 0.5 \\
\hline
\end{tabular}

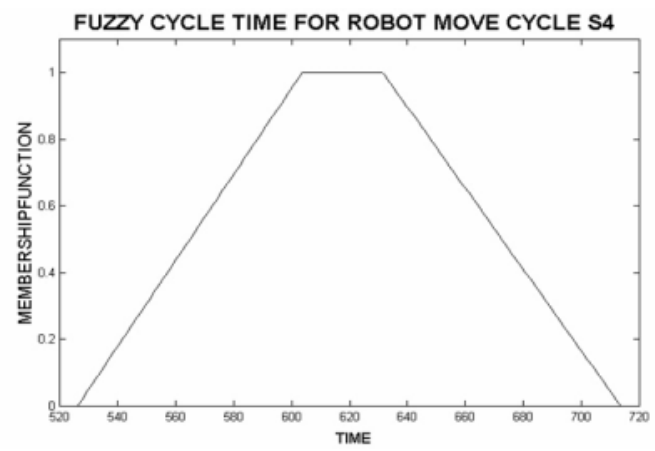

Fig. 15. fuzzy cycle time for robot move schedule $S_{4}$

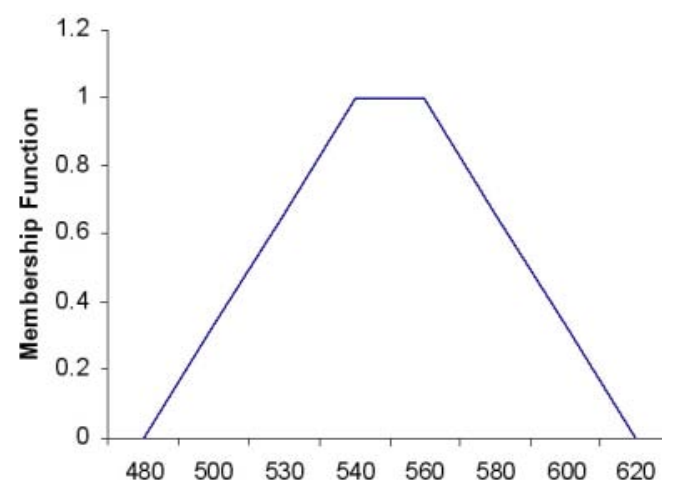

Fig. 16. Fuzzy cycle time for robot move cycle $S_{3}$

Using the same data of $M R C_{3}\left|k_{2}, S_{4}\right| C_{t}$ and Gilmore and Gomory algorithm that encoded in MATLAB software, the optimal schedule and it's related cycle time are shown as follows:

\begin{tabular}{|c|c|}
\hline Optimal sequence & Cycle time \\
\hline$Q^{*}=\{10,2,7,4,8,1,9,3,5,6\}$ & $C t^{*}=545.6$ \\
\hline
\end{tabular}

\section{Fuzzy problem:}

Again, using the same fuzzy data of $M R C_{3}\left|k \geq 2, S_{4}\right| C_{t}$, the optimal schedule that was generated by using fuzzy Gilmore and Gomory algorithm is: $Q^{*}=\{10,2,7,4,5,3,8,1,9,6\}$

The optimal fuzzy cycle is:

\begin{tabular}{|c|c|c|c|c|}
\hline & $\mathrm{a}$ & $\mathrm{B}$ & $\mathrm{c}$ & $\mathrm{d}$ \\
\hline$c_{t}$ & 472.9 & 532.25 & 557 & 620.1 \\
\hline
\end{tabular}

Fig. 16 demonstrates fuzzy cycle time of $S_{4}$. Using BADD defuzzification method with $\beta=100$, leads to defuzzified cycle time $D F\left(C_{t}\right)=544.48$ which is rather improved in comparison to the crisp results.

We also used the proposed algorithm for $S_{4}$ and $S_{5}$ and for both the fuzzy cycle times were rather improved with comparison with the crisp results. Moreover, we implemented the proposed algorithm for 100 scheduling problems and for all of them the results were better than the crisp ones.

\section{Conclusions}

This paper has addressed the scheduling of 2 and 3 machines robotic cells with fuzzy methodology. According to the Sethi et al. ${ }^{[25]}$ in two-machine robotic cell there exist two schedules that one of them can be solved easily and the second one is transferring to a solvable TSP. They state that three-machine robotic cells, there exist six schedules that one of them is easy, two of them are NP-Hard and three of them can be transferred into solvable TSP, using Gilmore and Gomory algorithm.

We generalized Gilmore and Gomory algorithm into Fuzzy Gilmore and Gomory one by using fuzzy numbers. Then, we tested and verified the algorithms in scheduling of an auto port supplier. We have solved 100 scheduling of this company and for all of them the result of fuzzy approach was better than the crisp ones.

There are some potential new works for this research: First, in three machine robotic cells, two schedules are NP-complete. Thus, we cannot use a contractive algorithm to solve these problems. In the next future, we will present fuzzy genetic algorithm and fuzzy simulated annealing to solve these problems. Moreover, we generated fuzzy numbers by using the knowledge of experts and tolerances of robot. A systematic algorithm is needed for this purpose. 


\section{References}

[1] R. G. Askin, K. S. Chiu. A graph partitioning procedure for machine assignment and cell formation, group technology. International Journal of Production Research, 1990, 28: 1555-1572.

[2] W. R. V. Baumann, J. Heansler, R. P. Hartmann, A. G. Stevens. Operating and idle times for cyclic multi-machine servicing. The Industrial Robot, 1981, 44-49.

[3] J. L. Burbidge. the Introduction of Group Technology. John Wiley Sons, New York, 1987.

[4] H. M. Chan, D. A. Milner. Direct clustering algorithm for group formation in cellular manufacturing. Journal of Manufacturing Systems, 1982, 1: 64-76.

[5] B. H. Cloybourn, J. R. Hewitt. Simularion of activity cycles for robot served manufacturing cells. Proceeding of the 1st International Conference on Flexible Manufacturing Systems, 1982, 443-452.

[6] Z. Drezner, S. Y. Nof. Optimal bin piching and insertion plans for assembly robots. IIE Transactions, 1998, 16: 262-270.

[7] P. C. Gilmore, R. E. Gomory. Sequencing a one state-variable machine: A solvable case of the traveling salesman problem. Operations research, 1964.

[8] R. L. Graham, E. L. Lawler, J. K. Lenstra, A. H. G. R. Kan. Optimization and approximation in deterministic sequencing and scheduling: A survey. Annals of Discrete Math, 1979, 5: 287-326.

[9] N. G. Hall, C. S. H. Kamoun. Scheduling in robotic cells: Classification, two and three machine cells. Operations Research, 1997, 45(3): 421-439.

[10] N. G. Hall, H. Kamoun, C. Sriskandarajah. Scheduling in robotics cells: Complexity and steady-state analysis. European journal of Operational Research, 1998, 109: 43-65.

[11] N. G. Hall, H. Kamoun, C. Sriskandrajah. Scheduling in robotic cells: Complexity and steady state analysis. 1995, 21-95. Department of Industrial Engineering, University of Toronto.

[12] J. Hartley. Robots at work. 1983. North-Holland Publishing Company.

[13] H. Kamoun. Scheduling in repetitive manufacturing system: Complexity, heuristics algorithm and system design. Ph.D Thesis, 1994. Department Of Industrial Engineering, University Toronto, Toronto, Canada.

[14] J. R. King, A. S. Spachis. Heuristics flow shop scheduling. Int.J.Prod.R., 1980, 18(3): 345-357.

[15] H. Kise, T. Shioyama, T.Ibaraki. Automated two machines flow shop scheduling: A solvable case. IIE Trans, 1991, 23(1): 10-16.

[16] A. S. Kondoleon. Cycle time analysis of robot assembly systems. Proceedings of the 9th Symposium on Industrial Robots, 1979, 575-587.

[17] A. Kusiak. The generalized group technology concept. International Journal of Production Research, 1987, 25: 56-569.

[18] R. Longendran, C. Sriskandarajah. Sequencing of robot activities and parts in two machine robotic cells. Int. J.Prod, Res., 1996.

[19] J. McCauley. Machine grouping for efficient production. the Production Engineer, 1972, 51: 3-7.

[20] S. Nof, Z. Drezner. Part flow in the robotic assembly plan problem, robotics and material flow. Elsevier, 1986. Ed.S.Y.Nof.

[21] Y. D. Noh, , B. Herring. Simulation model for an industrial robotic manufacturing cell. Int.J.Prod.Res., 1988, 26: $63-79$.

[22] G. R. Obinowits, A. mehrez, S. Samaddar. A scheduling model for multirobot assembly cells. International Journal of Flexible Manufacturing systems, 1991, 149-180.

[23] R. Rajagopalan., J. L. Barta. Design of cellular production systems: a graph theoretic approach. Int.Jour.Prod.Res, 1975, 13: 567-579.

[24] H. Seifoddini, P. M. Wolfe. Application of the simulating coefficient method in group technology. IIE Transactions, 1986, 18: 271-277.

[25] P. C. Sethi, S. Sriskandarajah, G. Sorger, J. Blazewicz, W. Kubiak. Sequencing of parts and robot moves in a robotic cell. International Journal of Flexible Manufacturing Systems, 1992, 4: 331-358.

[26] W. E. Wilhelm. Complexity of sequencing tasks in assembly cells attended by one or two robots. oval Research logistics, 1987, 34: 721-738.

[27] W. E. Wilhelm, S. C. Sarin. A structure for sequencing robot activities in machine loading applications. Int.J.Prod, Red., 1985, 23: 47-64.

[28] R. Yager, D. P. Filev. Slide: A simple adaptive defuzzification method. IEEE Transaction on Fuzzy Systems, 1993, 1(1): 69-78. 